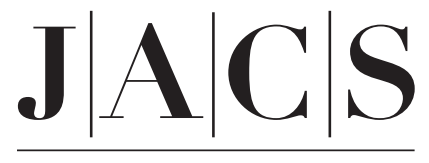

A R T I C L E S

Published on Web 06/03/2005

\title{
Evidence for the Isomerization and Decarboxylation in the Photoconversion of the Red Fluorescent Protein DsRed
}

\author{
Satoshi Habuchi, ,+,,\# Mircea Cotlet, ${ }^{\dagger, \nabla}$ Thomas Gensch, ${ }^{\ddagger}$ Teresa Bednarz,, \\ Sabina Haber-Pohlmeier, $\$$ Jef Rozenski," Gunter Dirix, ${ }^{\perp}$ Jan Michiels, ${ }^{\perp}$ \\ Jos Vanderleyden, ${ }^{\perp}$ Joachim Heberle, ${ }^{\S}$ Frans C. De Schryver,${ }^{\dagger}$ and \\ Johan Hofkens ${ }^{*}, t, O$ \\ Contribution from the Department of Chemistry, Katholieke Universiteit Leuven, \\ Celestijnenlaan 200F, 3001 Heverlee, Belgium, Institute for Biological Information Processing, \\ IBI-1, Research Centre Jülich, D-52425 Jülich, Germany, Institute for Biological Information \\ Processing, IBI-2, Research Centre Jülich, D-52425 Jülich, Germany, Rega Institute for Medical \\ Research, Faculty of Pharmaceutical Sciences, Katholieke Universiteit Leuven, \\ Minderbroedersstraat 10, 3000 Leuven, Belgium, Centre of Microbial and Plant Genetics, \\ Kasteelpark Arenberg 20, 3001 Heverlee, Belgium, and Unité CMAT, Université Catholique de \\ Louvain, Bâtiment Lavoisier Place L. Pasteur 1, 1348 Louvain-la-Neuve, Belgium \\ Received May 20,2004; E-mail: johan.hofkens@chem.kuleuven.ac.be; satoshi_habuchi@hms.harvard.edu

\begin{abstract}
Recently, it has been shown that the red fluorescent protein DsRed undergoes photoconversion on intense irradiation, but the mechanism of the conversion has not yet been elucidated. Upon irradiation with a nanosecond-pulsed laser at $532 \mathrm{~nm}$, the chromophore of DsRed absorbing at $559 \mathrm{~nm}$ and emitting at $583 \mathrm{~nm}$ (R form) converts into a super red (SR) form absorbing at $574 \mathrm{~nm}$ and emitting at $595 \mathrm{~nm}$. This conversion leads to a significant change in the fluorescence quantum yield from 0.7 to 0.01 . Here we demonstrate that the photoconversion is the result of structural changes of the chromophore and one amino acid. Absorption, fluorescence, and vibrational spectroscopy as well as mass spectrometry suggest that a cis-to-trans isomerization of the chromophore and decarboxylation of a glutamate (E215) take place upon irradiation to form SR. At the same time, another photoproduct (B) with an absorption maximum at $386 \mathrm{~nm}$ appears upon irradiation. This species is assigned as a protonated form of the DsRed chromophore. It might be a mixture of several protonated DsRed forms as there is at least two ways of formation. Furthermore, the photoconversion of DsRed is proven to occur through a consecutive two-photon absorption process. Our results demonstrate the importance of the chromophore conformation in the ground state on the brightness of the protein as well as the importance of the photon flux to control/avoid the photoconversion process.
\end{abstract}

\section{Introduction}

Proteins from the family of the green fluorescent protein (GFP) are extensively used in molecular and cellular biology as genetically encoded fluorescent markers for monitoring protein expression, protein dynamics, and protein-protein interaction. ${ }^{1-3}$ Wild-type GFP (wt-GFP) and some of the GFP-

\footnotetext{
† Department of Chemistry, Katholieke Universiteit Leuven.

Institute for Biological Information Processing, IBI-1, Research Centre Jülich.

$\S$ Institute for Biological Information Processing, IBI-2, Research Centre Jülich.

"Rega Institute for Medical Research, Katholieke Universiteit Leuven.

${ }^{\perp}$ Centre of Microbial and Plant Genetics.

- Unité CMAT, Université Catholique de Louvain.

\# Present Address: Department of Biological Chemistry and Molecular Pharmacology, Harvard Medical School, 240 Longwood Avenue, SGM209, Boston, MA 02115.

$\nabla$ Present Address: Los Alamos National Laboratory, Bioscience Division, Michelson (B-4), Mail Stop J586, Los Alamos, NM 87545.

(1) Chalfie, M. Green Fluorescent Proteins, Properties, Applications and Protocols; Wiley-Liss, Inc: New York, 1998.

(2) Sullivan, K. F.; Kay, S. A. Green Fluorescent Protein; Academic Press: San Diego, CA, 1999.

(3) Tsien, R. Y. Annu. Rev. Biochem. 1998, 67, 509-544.
}

10.1021/ja047023o CCC: $\$ 30.25$ @ 2005 American Chemical Society like proteins show photoinduced modifications (photoconversion) of the absorption and/or emission properties on irradiation. ${ }^{4-7}$ Although photoconversion might be a drawback for a stable fluorescent probe, it can serve for dual color imaging. 4,5

Recently, a photoconversion mechanism was proposed for wt-GFP, where the chromophore is transformed from the protonated form to the deprotonated form upon irradiation with UV or visible light. ${ }^{8,9}$ van Thor et al. demonstrated ${ }^{8}$ that the conversion results from decarboxylation of a glutamate (E222) residue, which is in close vicinity to the chromophore ( $p$ hydroxybenzylidene-imidazolinone). The same mechanism is

(4) Marchant, J. S.; Stutzmann, G. E.; Leissring, M. A.; LaFerla, F. M.; Parker, I. Nat. Biotechnol. 2001, 19, 645-649.

(5) Ando, R.; Hama, H.; Yamamoto-Hino, M.; Mizuno, H.; Miyawaki, A. Proc Natl. Acad. Sci. U.S.A. 2002, 99, 12651-12656.

(6) Chudakov, D. M.; Belousov, V. V.; Zaraisky, A. G.; Novoselov, V. V. Staroverov, D. B.; Zorov, D. B.; Lukyanov, S.; Lukyanov, K. A. Nat. Biotechnol. 2003, 21, 191-194.

(7) Patterson, G. H.; Lippincott-Schwartz, J. Science 2002, 297, 1873-1877.

(8) van Thor, J. J.; Gensch, T.; Hellingwerf, K. J.; Johnson, L. N. Nat. Struct. Biol. 2002, 9, 37-41.

(9) Bell, A. F.; Stoner-Ma, D.; Wachter, R. M.; Tonge, P. J. J. Am. Chem. Soc. 2003, 125, 6919-6926. 
proposed for the photoinduced deprotonation of some GFP mutants. ${ }^{7}$ We recently reported ${ }^{10}$ the photoconversion of the red fluorescent protein DsRed from a coral of the Discosoma genus in which the chromophore absorbing/emitting at 559/583 nm (R) converts on irradiation with a pulsed laser at $532 \mathrm{~nm}$ into a red-shifted super red (SR) species, absorbing at $574 \mathrm{~nm}$ and weakly emitting at $595 \mathrm{~nm}$. The red chromophore is the matured form of a GFP-like precursor ${ }^{11}$ and is known to be a deprotonated species at neutral $\mathrm{pH}$. In the case of DsRed, photoconversion leads to a deprotonated form with a significantly lower fluorescent quantum yield, in contrast to the photoconversion product of wt-GFP and its mutants. ${ }^{7,8}$ In the present paper, we report on the mechanism of the photoconversion taking place in DsRed by means of detailed ensemble absorption, fluorescence, and vibrational spectroscopy as well as mass spectrometry. We demonstrate the importance of the conformation of the chromophore for the brightness of the fluorescent protein. We show that the conversion takes place through a consecutive absorption of two photons.

\section{Materials and Methods}

Purification and Sample Preparation of DsRed Protein. Details about the bacterial expression and purification procedures for DsRed were published elsewhere. ${ }^{12}$ The protein was stored in a phosphate buffer saline (PBS) solution (10 mM, pH 7.4, Sigma).

Spectroscopy and Photoconversion. Steady-state absorption and fluorescence spectroscopy were performed on a Perkin-Elmer Lambda 40 spectrophotometer and a Spex Fluorolog 1500 fluorimeter (Spex Industries), respectively. A $10^{-4} \mathrm{M}$ DsRed solution in a quartz cell (path length: $1 \mathrm{~mm}$ ) was irradiated with $532 \mathrm{~nm}$ light provided by a nanosecond frequency-doubled Nd:YAG laser (Quanta Ray, $10 \mathrm{~Hz}$, fwhm $=8 \mathrm{~ns}$, Spectra-Physics). The average integral power at the sample ranged from $80 \mathrm{~mW} / \mathrm{cm}^{2}$ (1 MW peak power) to $350 \mathrm{~mW} / \mathrm{cm}^{2}$ (4.4 MW peak power). Irradiation experiments with continuous wave (CW) light were performed by using the $568 \mathrm{~nm}$ light from an $\mathrm{Ar}-\mathrm{Kr}$ ion laser (Stabilite 2018-RM, Spectra-Physics) at a power of $200 \mathrm{~mW}$ with two different beam waists, resulting in beam sizes of $0.7 \mathrm{~cm}^{2}$ (corresponding to $285 \mathrm{~mW} / \mathrm{cm}^{2}$ ) and $0.005 \mathrm{~cm}^{2}$ (corresponding to 40 $\mathrm{W} / \mathrm{cm}^{2}$ ), respectively. During the irradiation experiments, absorption and fluorescence spectra were recorded. The recorded fluorescence spectra were corrected according to literature procedures. ${ }^{13}$ Picosecond laser-induced time-correlated single-photon counting (TCSPC) fluorescence spectroscopy ${ }^{14,15}$ (excitation at 543 and $580 \mathrm{~nm}$ ) was performed on solutions of $10^{-6} \mathrm{M}$ fresh DsRed and of $10^{-4} \mathrm{M}$ photoconverted DsRed in PBS, respectively.

Photoconversion Measurement under the Microscope. Confocal laser scanning microscopy images of $E$. coli cells transfected with DsRed were measured by using an inverted microscope (IX70, Olympus) with an oil immersion objective lens $(100 \times$, NA 1.4 , Olympus) and Fluoview FV500 program. The cells were dropped on a polylysine coated cover glass. The excitation source was a $488 \mathrm{~nm}$ light from a CW Ar ion laser (model 163-C1210, Spectra-Physics) at a power of $9 \mathrm{~kW} / \mathrm{cm}^{2}$ in the focus. The excitation light was directed on the sample through the objective lens. Fluorescence was collected

(10) Cotlet, M.; Hofkens, J.; Habuchi, S.; Dirix, G.; Van Guyse, M.; Michiels, J.; Vanderleyden, J.; De Schryver, F. C. Proc. Natl. Acad. Sci. U.S.A. 2001, 98, 14398-14403.

(11) Gross, L. A.; Baird, G. S.; Hoffman, R. C.; Baldridge, K. K.; Tsien, R. Y. Proc. Natl. Acad. Sci. U.S.A. 2000, 97, 11990-11995.

(12) Cotlet, M.; Hofkens, J.; Köhn, F.; Michiels, J.; Dirix, G.; Van Guyse, M.; Vanderleyden, J.; De Schryver, F. C. Chem. Phys. Lett. 2001, 336, 415423.

(13) Lakowicz, J. R. Principles of Fluorescence Spectroscop; Plenum Press: New York, 1986; pp 4999-5006.

(14) Hofkens, J. et al. J. Phys. Chem. B 2001, 105, 4999-5006.

(15) Maus, M.; Rousseau, E.; Cotlet, M.; Hofkens, J.; Schweitzer, G.; Van der Auweraer, M.; De Schryver, F. C.; Kruegen, A. Rev. Sci. Instrum. 2001, $72,36-40$. by the same objective, passed through a dichroic mirror (Q497LP, Chroma Technology), and separated into a green and a red emission by a dichroic mirror (DM560, Olympus). The green and the red emission were passed through filters (BA505-525, Chroma Technology, for the green emission, and BA560IF, Chroma Technology, for the red emission, respectively) and imaged, via a $100 \mu \mathrm{m}$ pinhole, into photomultiplier tubes. Photoconversion of DsRed was performed by irradiating a cell with a $543 \mathrm{~nm}$ light from a CW He-Ne laser (05LGP-193, Melles Griot) for $2 \mathrm{~s}$ at a power of $28 \mathrm{~kW} / \mathrm{cm}^{2}$ in the focus. A $543 \mathrm{~nm}$ dichroic mirror (Q555LP, Chroma Technology) was used for irradiation.

FT-IR Spectroscopy. FT-IR difference spectroscopy was performed on a Bruker IFS 66v spectrometer (Bruker Analytische Messtechnik). The preparation of the sample for the conventional transmission technique was as follows: a droplet of a DsRed solution $\left(10^{-3} \mathrm{M}\right)$ was put on a $\mathrm{BaF}_{2}$ cuvette and dried by a stream of nitrogen. A droplet of water was added, and the cuvette was sealed by a cover window of the same material (for more details, see ref 16). For sample illumination, the $532 \mathrm{~nm}$ light provided by a nanosecond frequency-doubled $\mathrm{Nd}$ : YAG laser (Quanta Ray, GCR $12 \mathrm{~S}, 10 \mathrm{~Hz}$, fwhm $=8 \mathrm{~ns}$, SpectraPhysics) at a power of $5 \mathrm{~mJ} /$ pulse was used. All data were collected with $2 \mathrm{~cm}^{-1}$ resolution. Temperature of the sample was set to $293 \mathrm{~K}$ by a circulating water bath.

Raman Spectroscopy. Raman spectra were aquired under preresonant conditions with excitation at $752 \mathrm{~nm}$ from a $\mathrm{Kr}$ ion laser (Innova 90K, Coherent). The laser radiation was fed into a confocal LabRam spectrometer (Jobin-Yvon), where the laser emission was focused by a microscope objective $(\times 10$, Olympus) to a spot (diameter approximately $20 \mu \mathrm{m}$ ) and an effective laser power of $50 \mathrm{~mW}$. The sample was prepared by gently concentrating the protein on the inner surface of a quartz cuvette. The light scattered from the sample was monitored in backscattering configuration, where the Rayleigh-scattered light is rejected by a holographic notch filter. The inelastically scattered photons are dispersed by a grating $(1800$ grooves $/ \mathrm{mm})$ and detected by a Peltier-cooled CCD camera. The resulting Raman spectrum, corrected by subtracting an apparatus-specific, wavenumber-dependent background, has a datapoint spacing of $0.5 \mathrm{~cm}^{-1}$ and a spectral resolution of $2 \mathrm{~cm}^{-1}$. The spectrum was measured with a collection time of $1 \mathrm{~h}$.

Mass Spectrometry. Electrospray mass spectra were acquired in positive ion mode on a quadrupole/orthogonal acceleration time-offlight mass spectrometer (Q-Tof 2, Micromass). Samples were infused with a flow rate of $5 \mu \mathrm{L} / \mathrm{min}$ in a mixture of acetonitrile and water (1:1) containing $1 \%$ acetic acid. Capillary voltage was set to $3000 \mathrm{~V}$, and a cone voltage of $35 \mathrm{~V}$ was used. The masses of the compounds were obtained by deconvolution of the spectra using the MaxEnt algorithm of the Masslynx 3.4 software. The accuracy of the mass is $\pm 1 \mathrm{Da}$.

\section{Results and Discussion}

Reaction Scheme of the Photoconversion. The visible absorption spectrum of DsRed shows two maxima located at 559 and $483 \mathrm{~nm}$ (see Figure 1a). ${ }^{17,18}$ The 559-nm absorption peak was assigned to the mature chromophore (R), while the absorption peak at $483 \mathrm{~nm}$ was attributed to an immature green precursor form of R. ${ }^{11}$ On irradiation with a 532-nm nanosecondpulsed laser, the absorption peak at $559 \mathrm{~nm}$ diminished and shifted bathochromically up to $574 \mathrm{~nm}$ (see Figure 1a). ${ }^{10}$ Concomitantly a new band appeared at $386 \mathrm{~nm}$. The absorption band associated with the immature green chromophore modified

(16) Heberle, J.; Zscherp, C. Appl. Spectrosc. 1996, 50, 588-596.

(17) Baird, G. S.; Zacharias, A. A.; Tsien, R. Y. Proc. Natl. Acad. Sci. U.S.A 2000, 97, 11984-11989.

(18) Matz, M. V.; Fradkov, A. F.; Labas, Y. A.; Savitsky, A. P.; Zaraisky, A G.; Markelov, M. L.; Lukyanov, S. A. Nat. Biotechnol. 1999, 17, 969973. 
neither in position nor in intensity. Two isosbestic points, at 343 and $467 \mathrm{~nm}$, were observed. The absorption spectra of DsRed were decomposed into four bands by using Gaussian functions: a blue (B) band peaking at around $386 \mathrm{~nm}$, a green (G) band peaking at around $479 \mathrm{~nm}$, a red (R) band peaking at around $559 \mathrm{~nm}$, and a super-red (SR) band peaking at around $574 \mathrm{~nm}$ (see Figure 1b). ${ }^{19}$ After resting in the dark for $24 \mathrm{~h}$, no modification was noticed in the absorption spectrum of the photoconverted DsRed. However, it should be mentioned that $\mathrm{SR}$ and $\mathrm{B}$ are not long-term stable species. The absorption of SR and B decreased substantially in the dark after a week. The time evolution of the peak absorbancies of the decomposed bands is depicted in Figure 1c. The absorbance of $\mathrm{R}$ decreased with increasing irradiation time at a given intensity; the absorbance of B increased with increasing irradiation time. The peak absorption of SR first increased, and after reaching a maximum value, it decreased again. This suggests that SR is an intermediate species. On the basis of the time evolution of $\mathrm{R}, \mathrm{SR}$, and $\mathrm{B}$, a consecutive reaction from $\mathrm{R}$ to $\mathrm{SR}$ and finally to $\mathrm{B}$ might be applied as a kinetic model of the photoconversion. We excluded the contribution of photobleaching of the chromophore, since the positions of the isosbestic points were constant during the measurement. The time evolution of the three species, however, could not be fitted with a consecutive model (Figure 9, see Supporting Information). Consequently, the time evolution of the peak absorbencies was analyzed with a model that takes into account, in addition to the consecutive reaction from $\mathrm{R}$ to $\mathrm{SR}$ to $\mathrm{B}$, direct conversion from $\mathrm{R}$ to $\mathrm{B}$. In this model, the time evolution of the peak absorbencies of $\mathrm{R}, \mathrm{SR}$, and $\mathrm{B}$ can be described as follows,

$$
\mathrm{Abs}_{\mathrm{R}}=a_{1} \exp \left\{\left(-k_{1}-k_{3}\right) t\right\}
$$

$$
\begin{aligned}
& \mathrm{Abs}_{\mathrm{SR}}= \\
&-\left(\frac{k_{1} a_{2}}{k_{2}-k_{1}-k_{3}}\right) \exp \left(-k_{2} t\right)\left[1-\exp \left\{\left(k_{2}-k_{1}-k_{3}\right) t\right\}\right] \\
& \mathrm{Abs}_{\mathrm{B}}=a_{3}\left[1-\left(\frac{k_{2}-k_{3}}{k_{2}-k_{1}-k_{3}}\right)\right. \exp \left\{\left(-k_{1}-k_{3}\right) t\right\}+ \\
&\left.\left(\frac{k_{1}}{k_{2}-k_{1}-k_{3}}\right) \exp \left(-k_{2} t\right)\right]
\end{aligned}
$$

where $k_{1}$ and $k_{2}$ are the rate constants of the photoconversion from $\mathrm{R}$ to $\mathrm{SR}$ and from $\mathrm{SR}$ to $\mathrm{B}$, respectively. $k_{3}$ is the rate constant of the photoconversion of R into B. $a_{1}, a_{2}$, and $a_{3}$ are the concentration of the initial species $(\mathrm{R})$ multiplied by the extinction coefficient $(\epsilon)$ of R, SR, and B, respectively. Fitting the time evolution of $\mathrm{R}, \mathrm{SR}$, and $\mathrm{B}$ by eqs 1,2 , and 3 , rate constants of $2.1 \times 10^{-3} \mathrm{~s}^{-1}\left(k_{1}\right), 2.2 \times 10^{-4} \mathrm{~s}^{-1}\left(k_{2}\right)$, and 4.4 $\times 10^{-4} \mathrm{~s}^{-1}\left(k_{3}\right)$, respectively, were obtained (see Figure 1c). The $a$ values obtained from the fitting were $0.61\left(a_{1}\right), 0.40\left(a_{2}\right)$, and $0.12\left(a_{3}\right)$. The difference in the $a$ values is indicative of the different $\epsilon$ of the three species (R, SR, B). From these $a$ values, the $\epsilon$ is estimated to be $49000 \mathrm{M}^{-1} \mathrm{~cm}^{-1}$, and 15000 $\mathrm{M}^{-1} \mathrm{~cm}^{-1}$ for SR and $\mathrm{B}$, respectively, assuming the $\epsilon$ of $\mathrm{R}$ to be $75000 \mathrm{M}^{-1} \mathrm{~cm}^{-1} 17$ (see Figure 1b). According to the analysis of the time evolution of the absorption spectrum, a

(19) The absorption spectra of DsRed were decomposed into four bands assuming that the $\mathrm{R}$ form was completely converted into the SR form after $93 \mathrm{~min}$ of irradiation with a 532-nm pulsed laser light at an excitation power of $350 \mathrm{~mW}$
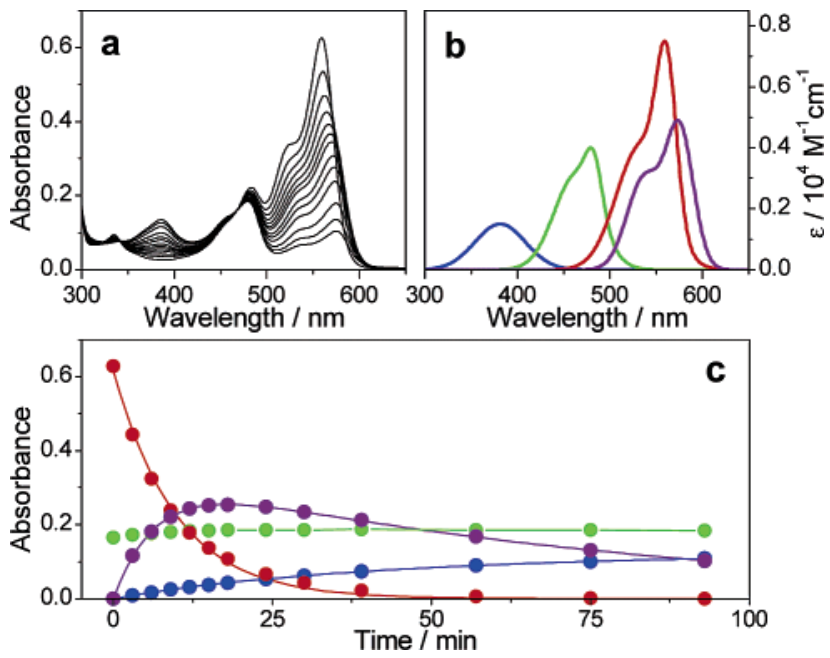

Figure 1. (a) Time evolution of the absorption spectrum of DsRed in PBS on irradiation with a $532-\mathrm{nm}$ pulsed laser at an excitation power of 350 $\mathrm{mW} / \mathrm{cm}^{2}$. (b) Decomposed absorption spectra of B (blue line), G (green line), $\mathrm{R}$ (red line), and SR (purple line). The extinction coefficients of B and SR are estimated from the $a$ values obtained by eqs 2 and 3, respectively. Note that the absorption spectrum of $\mathrm{G}$ is drawn in an arbitrary unit. (c) Time evolution of the peak absorbances of B (blue circle), G (green circle), $\mathrm{R}$ (red circle), and SR (purple circle) during the irradiation experiment. These time evolutions of the peak absorbances are fitted by eq 1 for $\mathrm{R}$, eq 2 for SR, and eq 3 for B, and the fitting curves are shown as the solid lines.

reaction scheme of the photoconversion can be described as a competitive reaction between direct conversion from $\mathrm{R}$ to $\mathrm{B}$ and consecutive reaction from $\mathrm{R}$ to $\mathrm{SR}$ to $\mathrm{B}$.

Spectroscopic and Chemical Nature of SR and B. The fluorescence spectrum of fresh (nonirradiated) DsRed displays a maximum at $583 \mathrm{~nm}$ independent of the excitation wavelength (see Figure 2a, b). ${ }^{17,18}$ An additional peak, weak in intensity and located at around $500 \mathrm{~nm}$, is present, when DsRed is excited at $470 \mathrm{~nm}$, which corresponds to the absorption band of $\mathrm{G}$ (see Figure 2a). The 500 and $583 \mathrm{~nm}$ emission peaks were assigned to $\mathrm{R}$ and $\mathrm{G}$, respectively. DsRed is known to form a tetramer even at nanomolar concentration. The observed red emission $(583 \mathrm{~nm}) \mathrm{can}$, therefore, be interpreted by resonance energy transfer from $\mathrm{G}$ to $\mathrm{R} .{ }^{10} \mathrm{On}$ irradiation with a nanosecond-pulsed laser at $532 \mathrm{~nm}$, the fluorescence spectrum (470 nm excitation) of the red emission decreased and shifted bathochromically to $595 \mathrm{~nm}$ (see Figure 2a, inset). Simultaneously, the green emission $(500 \mathrm{~nm})$ revealed a 2.5 -fold intensity enhancement (see Figure $2 \mathrm{a}$ and inset). While the fresh sample showed a ratio of the green to red emission of 0.11 on irradiation for $93 \mathrm{~min}$, this ratio increased to 17 , about 160 times larger than the original value. The fluorescence spectrum excited at the absorption band of R (543 nm) showed an analogous decrease and bathochromic shift for the red emission $(583 \mathrm{~nm}$ ) (see Figure 2b) upon irradiation. ${ }^{10}$ The time evolution of the maximum fluorescence intensity on irradiation $(532 \mathrm{~nm})$ displayed a similar behavior as observed for the absorption band of $\mathrm{R}$ (see Figure $2 \mathrm{~b}$ inset), suggesting that the emission of $\mathrm{R}$ is dominantly contributing to the detected red emission. Indeed, the fluorescence quantum yield $(\Phi)$ of SR is calculated to be 0.012 , i.e., $\sim 60$ times smaller than that of $\mathrm{R}(\Phi=0.7)$, taking into account the difference in the absorbance for the fresh and photoconverted samples at the excitation wavelength together with the 250 times smaller fluorescence intensity of the photoconverted DsRed (93 min irradiation) as compared to the fresh sample. 

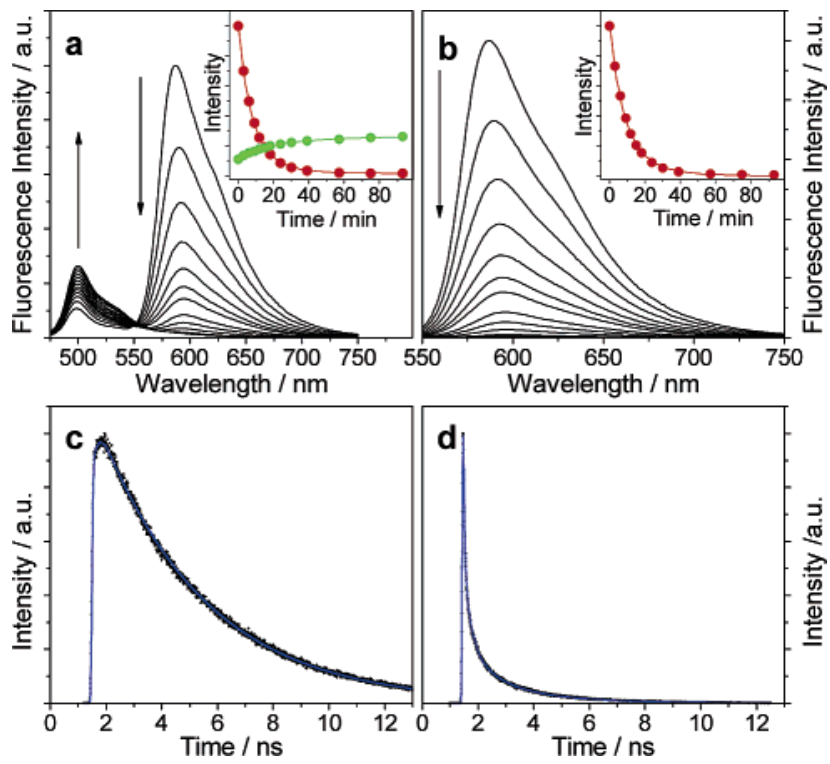

Figure 2. (a) Time evolution of the fluorescence spectrum of DsRed in PBS excited at $470 \mathrm{~nm}$ upon irradiation with a 532-nm pulsed laser at an excitation power of $350 \mathrm{~mW} / \mathrm{cm}^{2}$. (Inset) Time evolution of the peak intensity of the red emission (red circle) and green emission (green circle) (b) Time evolution of the fluorescence spectrum of DsRed in PBS excited at $543 \mathrm{~nm}$ during irradiation with a $532 \mathrm{~nm}$ pulsed laser at an excitation power of $350 \mathrm{~mW} / \mathrm{cm}^{2}$. (Inset) Time evolution of the peak intensity of the red emission. (c) Fluorescence decay (dot) of the fresh DsRed excited at $543 \mathrm{~nm}$ and detected at $600 \mathrm{~nm}$. The blue line shows the fitting curves. (d) Fluorescence decay (dot) of the photoconverted DsRed excited at $543 \mathrm{~nm}$ and detected at $600 \mathrm{~nm}$. The blue line shows the fitting curves.

The fluorescence of the fresh DsRed excited at $543 \mathrm{~nm}$ decays single exponentially with a decay time constant of $3.6 \mathrm{~ns},{ }^{10,20}$ independent of the monitoring wavelength (see Figure 2c). In contrast to the fresh sample, the fluorescence decay detected from 600 up to $660 \mathrm{~nm}$ on excitation of the photoconverted DsRed (93 min irradiation) at $580 \mathrm{~nm}$ displays a multiexponential behavior (see Figure 2d). Four components, having the decay times of $2.8,0.66,0.13$, and 0.03 ns with the relative amplitude of $0.05,0.08,0.24$, and 0.64 , were necessary to globally fit the decays. The low fluorescence quantum yield and the fast decay time suggest that SR has additional nonradiative deactivation channels as compared to R. These observations suggest that the R and SR chromophore have different structures and/or different protein environments. Hence we next investigated the chemical structure of the chromophore by FT-IR as well as Raman spectroscopy.

Negative and positive bands in a difference FT-IR spectrum correspond to vibrations of the nonirradiated and irradiated protein samples, respectively (see Figure 3a). Bands in the Raman spectrum, which has been taken in preresonance mode, can be attributed to vibrations of the chromophore of nonirradiated DsRed (see vertical lines in Figure 3a). Most of the negative peaks in the difference FT-IR spectrum align with the vibrational bands of the chromophore observed in the Raman spectrum. These findings suggest that the structural change upon photoconversion is dominated by changes in the chromophore structure.

The largest feature in the FT-IR difference spectrum at the early stage of the photoconversion is a negative/positive band

(20) Heikal, A. A.; Hess, S. T.; Baird, G. S.; Tsien, R. Y.; Webb, W. W. Proc. Natl. Acad. Sci. U.S.A. 2000, 97, 11996-12001.
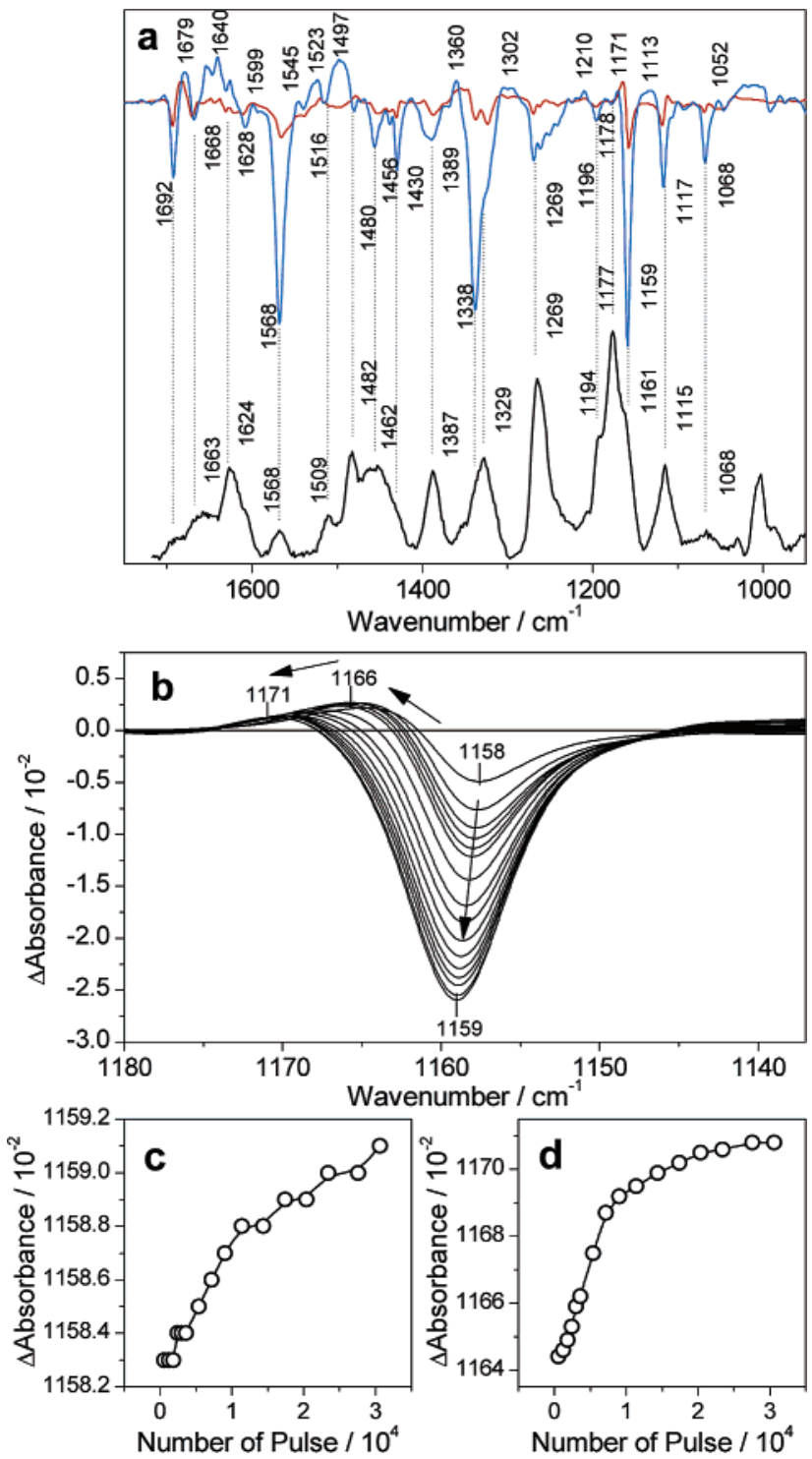

Figure 3. (a) Upper spectra show light-induced FT-IR difference spectra of DsRed in PBS after 600 (red line) and 30600 (blue line) laser pulses with a 532-nm pulsed laser. Lower spectrum shows Raman spectrum of DsRed in BPS. (b) Time evolution of the FT-IR spectrum of DsRed at around $1160 \mathrm{~cm}^{-1}$ upon irradiation with a 532-nm pulsed laser. (c) Time evolution of the peak position of the $1158 \mathrm{~cm}^{-1}$ band. (d) Time evolution of the peak position of the $1166 \mathrm{~cm}^{-1}$ band.

pair at $1158 \mathrm{~cm}^{-1}$ (negative) $/ 166 \mathrm{~cm}^{-1}$ (positive) (see Figure $3 \mathrm{a}, \mathrm{b})$. After the complete photoconversion, the band pair shifted to $1159 \mathrm{~cm}^{-1}$ (negative)/1171 $\mathrm{cm}^{-1}$ (positive) (see Figure 3a). The temporal evolution of the difference spectrum in this region shows that, for the first few hundred applied laser pulses, the absorption is rising at $1166 \mathrm{~cm}^{-1}$ while decreasing at $1158 \mathrm{~cm}^{-1}$ (see Figure $3 b$ ). At later times of the photoconversion at both frequencies, a decrease of the infrared absorption is observed concomitant with a shift to higher wavenumbers for the positive band (see Figure $3 b$ ). The spectral shifts can be clearly seen in Figure $3 \mathrm{c}$ and $\mathrm{d}$. These features are interpreted by a two-step process. According to the time evolution of the absorption spectrum of DsRed upon irradiation, the first and the second steps correspond to the conversion from $\mathrm{R}$ to $\mathrm{SR}$ and the conversion from R or SR to B, respectively.

The bands at $1158 \mathrm{~cm}^{-1}$ (negative)/1166 $\mathrm{cm}^{-1}$ (positive) have been identified as marker bands for the trans-to-cis isomerization 
Scheme 1. Mechanism for the Photoconversion from $\mathrm{R}$ to $\mathrm{SR}$ in DsRed

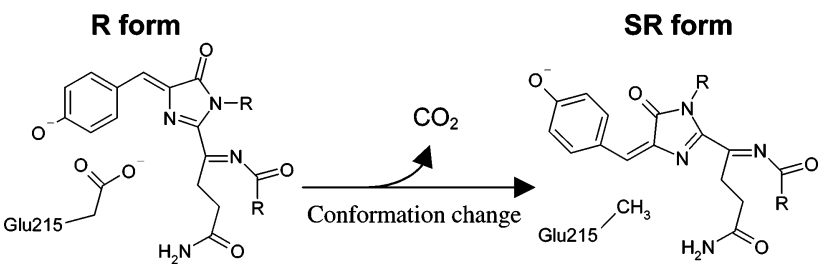

in the chromophore of photoactive yellow protein, ${ }^{21-23}$ which owes the same phenol group + double bond structure as GFP and DsRed. Other less pronounced marker bands for the isomerization ${ }^{23,24}$ cannot be clearly observed in the spectrum. Nevertheless, it can be deduced from the assignment of the feature at $1158 \mathrm{~cm}^{-1}$ (negative) $/ 1166 \mathrm{~cm}^{-1}$ (positive), that the photoconversion from $\mathrm{R}$ to $\mathrm{SR}$ could be the result of a cis-totrans photoisomerization of the chromophore as shown in Scheme 1.

The strong negative band at $1159 \mathrm{~cm}^{-1}$ with a weak counterpart at $1171 \mathrm{~cm}^{-1}$ observed at a later stage of the photoconversion has been assigned as a marker band for the protonation reaction of GFP from Aequoria victoria ${ }^{25,26}$ and DsRed. ${ }^{27}$ Therefore, the photoconversion from R or SR to B can be attributed to protonation of the chromophore. The absorption spectrum of $\mathrm{B}$ displays an absorption maximum at $386 \mathrm{~nm}$ (see Figure 1b) which is close to the absorption peak of the protonated form of the DsRed chromophore, ${ }^{28,29}$ supporting this idea. Whether the B form of DsRed has undergone complete cis-to-trans photoisomerization cannot be decided based on the FT-IR data. No change in the shape of the absorption spectrum of $\mathrm{B}$ was observed during the conversion which might indicate that the B chromophore directly converted from $\mathrm{R}$ also has a trans configuration.

Another important feature in the difference FT-IR spectrum is the pair of bands at 1568 and $1389 \mathrm{~cm}^{-1}$ (see Figure 3a). These bands are present in both the early and later stages of the conversion and can be tentatively assigned. The negative band at $1568 \mathrm{~cm}^{-1}$ represents contributions from three different vibrations, a vibration of the phenolic group, ${ }^{30}$ a vibration delocalized over the imidazolinone ring with significant $v(\mathrm{C}=$ N) character, ${ }^{30}$ and an asymmetric vibration of a $\mathrm{COO}^{-}$group. A complementary symmetric stretch of the $\mathrm{COO}^{-}$group is observed at $1389 \mathrm{~cm}^{-1}$. The parallel disappearance of these two bands $\left(1568 \mathrm{~cm}^{-1}, 1389 \mathrm{~cm}^{-1}\right)$ can be interpreted as the loss of a $\mathrm{COO}^{-}$group, i.e., a decarboxylation of an amino acid residue containing a $\mathrm{COO}^{-}$in the side chain.

The only vibrations of amino acids showing infrared absorption in the region above $1700 \mathrm{~cm}^{-1}$ are the $\mathrm{C}=\mathrm{O}$ stretching

(21) Brudler, R.; Rammelsberg, R.; Woo, T. T.; Getzoff, E. D.; Gerwert, K. Nat. Struct. Biol. 2001, 8, 265-270.

(22) Imamoto, Y.; Shirahige, Y.; Tokunaga, F.; Kinoshita, T.; Yoshihara, K.; Kataoka, M. Biochemistry 2001, 40, 8997-9004.

(23) Groot, M. L.; van Wilderen, L. J. G. W.; Larsen, D. S.; van der Horst, M. A.; van Stokkum, I. H. M.; Hellingwerf, K. J.; van Grondelle, R. Biochemistry 2003, 42, 10054-10059.

(24) Haker, A.; Hendriks, J.; van Stokkum, I. H. M.; Heberle, J.; Hellingwerf, K. J.; Crielaard, W.; Gensch, T. J. Biol. Chem. 2003, 278, 8442-8451.

(25) Bell, A. F.; He, X.; Wachter, R. M.; Tonge, P. J. Biochemistry 2000, 39, $4423-4431$

(26) van Thor, J. J.; Pierik, A. J.; Nugteren-Roodzant, A.; Xie, A.; Hellingwerf, K. J. Biochemistry 1998, 37, 16915-16921.

(27) He, X.; Bell, A. F.; Tonge, P. J. Org. Lett. 2002, 4, 1523-1526.

(28) Vrzheshch, P. V.; Akovbian, N. A.; Varfolomeyev, S. D.; Verkhusha, V. V. FEBS Lett. 2000, 487, 203-208.

(29) Wiehler, J.; von Hummel, J.; Steipe, B. FEBS Lett. 2001, 487, 384-389.

(30) He, X.; Bell, A. F.; Tonge, P. J. J. Phys. Chem. B 2002, 106, 6056-6066.

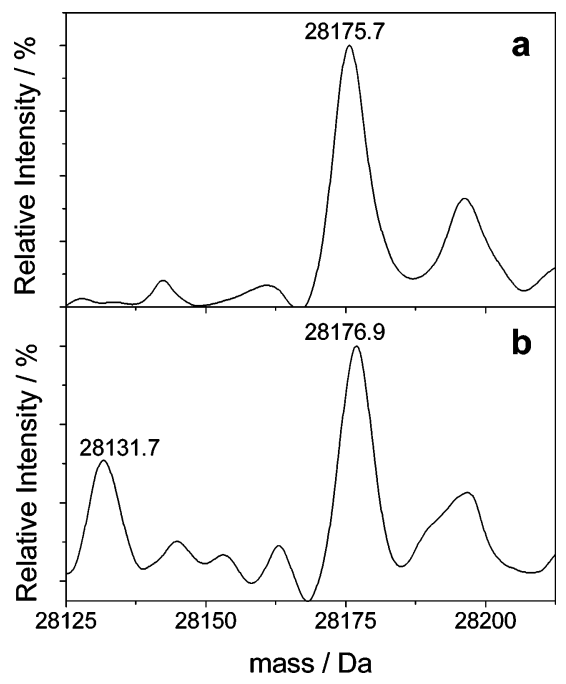

Figure 4. Mass spectra of (a) fresh DsRed and (b) photoconverted DsRed irradiated with a 532-nm nanosecond-pulsed laser.

modes of $\mathrm{COOH}$ groups. No band can be observed above 1700 $\mathrm{cm}^{-1}$ in the FT-IR difference spectra during the photoconversion. It can therefore be concluded that no change in absolute number or environment of a $\mathrm{COOH}$ group occurs during the DsRed photoconversion. It rather indicates that decarboxylation of glutamate, most probably at the E215 residue (vide infra) which is in close vicinity of the chromophore, takes place during the photoconversion (see Scheme 1) with E215 in its deprotonated form $\left(\mathrm{COO}^{-}\right)$.

This idea is further supported by mass spectrometry measurements. The mass spectrum of fresh DsRed revealed only one species with a mass of $28176 \mathrm{Da}$, which corresponds well with a calculated value $(28178 \mathrm{Da})$ of the protein mass including His-tags (see Figure 4a).

In contrast to fresh DsRed, the mass spectrum of the DsRed sample photoconverted with a nanosecond-pulsed $532 \mathrm{~nm}$ laser showed two peaks with masses of 28176 and 28132 Da which can be attributed to the mass of $\mathrm{R}$ and SR, respectively (see Figure $4 \mathrm{~b}$ ). The fact that the mass of SR is $44 \mathrm{Da}$ smaller than that of R together with the results of the FT-IR measurement strongly suggests that the decarboxylation reaction takes place during the photoconversion from $\mathrm{R}$ to SR. The presence of both peaks with masses of 28176 and 28132 Da even after prolonged irradiation can be explained by the presence of a substantial fraction of the green precursor form or by an incomplete decarboxylation of the SR form of DsRed.

As mentioned above, SR has an additional nonradiative deactivation channel as compared to $\mathrm{R}$. It is proposed for asCP, a GFP-like nonfluorescent protein that becomes bright upon irradiation, that trans-to-cis isomerization of the chromophore might be the origin of the change in the fluorescence quantum yield. ${ }^{31}$ Since the chromophore in the SR state is suggested to have a trans configuration, the dimness of SR might arise from its trans configuration. However, a recent X-ray crystallographic study revealed that the chromophore of a red fluorescent protein eqFP611, which has the same chemical structure as DsRed, has a trans configuration, ${ }^{32}$ although the protein has relatively high

(31) Chudakov, D. M.; Feofanov, A. V.; Mudrik, N. N.; Lukyanov, S.; Lukyanov, K. A. J. Biol. Chem. 2003, 278, 7215-7219.

(32) Petersen, J.; Wilmann, P. G.; Beddoe, T.; Oakley, A. J.; Devenish, R. J.; Prescott, M.; Rossjohn, J. J. Biol. Chem. 2003, 278, 44626-44631. 


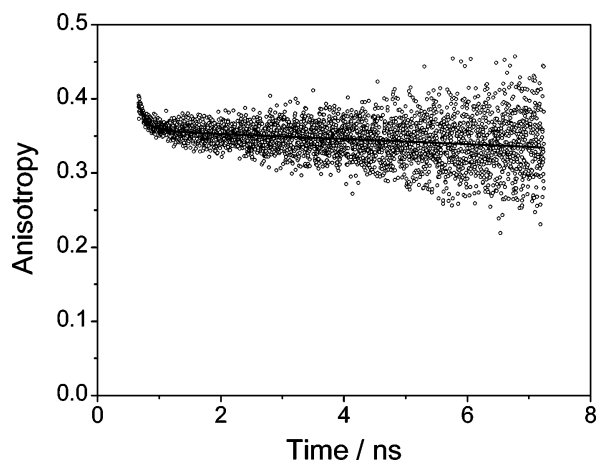

Figure 5. Fluorescence anisotropy decay (dot) of the photoconverted DsRed excited at $580 \mathrm{~nm}$ and detected at $640 \mathrm{~nm}$. The solid line shows the fit by using a double-exponential decay function.

fluorescence quantum yield $(\Phi=0.45) \cdot{ }^{33}$ A more plausible interpretation for the dimness of SR is the torsional motion of the SR chromophore around the two central bonds in the excited state. Theoretical calculations suggested that the twisting of the chromophore in the excited state decreases the energy gap between the excited and ground state potential energy surface, resulting in the nonradiative deactivation of the excited state. ${ }^{34}$ For an efficient torsional motion, the activation energy should be low, ${ }^{35}$ meaning that the hydrogen-bonding network around the chromophore and the free volume around the chromophore play an important role. Therefore, it is most likely that the hydrogen-bonding network around the chromophore in the SR state of DsRed is substantially different from that in the R state due to its proposed trans configuration and the decarboxylation of E215, since the carboxylate of E215 fills the adjacent space near to the phenolic group of the chromophore. ${ }^{36}$ Therefore, the efficient nonradiative deactivation of SR might be attributed to the different hydrogen-bonding network as well as the free volume around the chromophore, which allow the formation of the twisted configuration. Free rotation of the chromophore as the origin of the dimness of SR, however, is excluded, since SR shows similar high anisotropy and long rotational correlation times compared to $\mathrm{R}$ in time-resolved anisotropy measurement (see Figure 5).

The photoconversion from $\mathrm{R}$ to $\mathrm{SR}$ form was also observed at the single-molecule level. ${ }^{9}$ The fluorescence of SR form is, however, very unlikely to be detected by the single-molecule measurement because of the very low fluorescence quantum yield of SR form $(\Phi=0.012)$. From our experience, the fluorescence quantum yield of a molecule should be higher than 0.1 to be detected at the single-molecule level. At the moment, we can only speculate on the origin of the enhancement of the fluorescence in the single-molecule measurements. One possibility is matrix-induced fluorescence enhancement. The conformation of the chromophore or the interaction between the chromophore and the barrel might be modified by fixing the molecule in a polymer matrix. Because the chromophore conformation and the free volume around the chromophore are crucial for the fluorescence properties of DsRed as discussed

(33) Wiedenmann, J.; Schenk, A.; Röcker, C.; Girod, A.; Spindler, K.-D.; Nienhaus, G. U. Proc. Natl. Acad. Sci. U.S.A. 2002, 99, 11646-11651.

(34) Weber, W.; Helms, V.; McCammon, J. A.; Langhoff, P. W. Proc. Natl. Acad. Sci. U.S.A. 1999, 96, 6177-6182

(35) Follenius-Wund, A.; Bourotte, M.; Schmitt, M.; Iyice, F.; Lami, H.; Bourguignon, J.-J.; Haiech, J.; Pigault, C. Biophys. J. 2003, 85, 18391850 .

(36) Yarbrough, D.; Wachter, R. M.; Kallio, K.; Matz, M. V.; Remington, S. J. Proc. Natl. Acad. Sci. U.S.A. 2001, 98, 462-467.

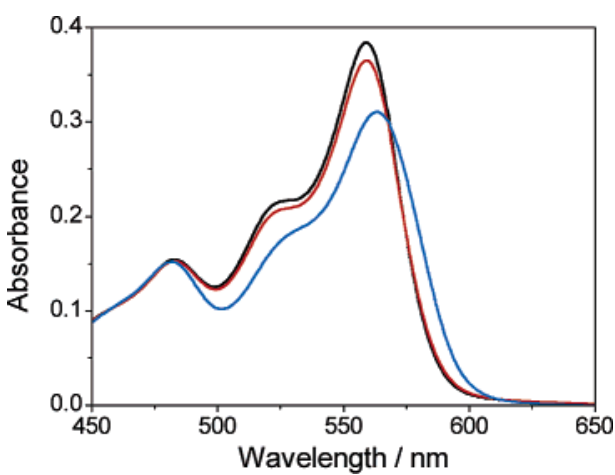

Figure 6. Changes in the absorption spectrum of DsRed on irradiation with a $568 \mathrm{~nm} \mathrm{cw}$ laser at the irradiation power of $200 \mathrm{~mW}$. Absorption spectrum before irradiation (black line). Absorption spectra after $330 \mathrm{~min}$ irradiation with the beam size of $0.7 \mathrm{~cm}^{2}\left(300 \mathrm{~mW} / \mathrm{cm}^{2}\right.$, red line $)$ and 0.005 $\mathrm{cm}^{2}\left(40 \mathrm{~W} / \mathrm{cm}^{2}\right.$, blue line), respectively.

above, such the conformation change induced by a matrix can influence the fluorescence properties of the chromophore significantly. The influence of a polymer matrix has also been observed for the fluorescent protein HcRed. ${ }^{37}$

Mechanism of the Photoconversion. To investigate the influence of the photon flux on the photoconversion, irradiation measurements with different photon densities were performed by using a $568-\mathrm{nm}$ laser. Figure 6 shows the changes in the absorption spectrum of DsRed on irradiation at the same power $(200 \mathrm{~mW})$ and same irradiation time, but with different beam size.

When irradiated with a wide beam, corresponding to $300 \mathrm{~mW} /$ $\mathrm{cm}^{2}$, almost no photoconversion was observed (see Figure 6). On the other hand, when the sample was irradiated with a focused beam, corresponding to $40 \mathrm{~W} / \mathrm{cm}^{2}$, the absorption spectrum displayed a red shift up to $563 \mathrm{~nm}$ (after $330 \mathrm{~min}$ irradiation; a further shift to $566 \mathrm{~nm}$ was observed after 600 $\mathrm{min}$ ), similar to that observed for $532 \mathrm{~nm}$ nanosecond-pulsed laser irradiation (see Figure 6). These findings clearly demonstrated that the photoconversion cannot be described as a simple one-photon excitation process. The results rather indicate that the photoconversion occurs through a consecutive two-photon excitation mechanism. A short-lived intermediate is formed after the absorption of the first photon, and the converted species (SR) is produced by excitation of that intermediate by the second absorbed photon. It is calculated from the absorption cross section $(\sigma)$ of DsRed at the irradiated wavelength $(568 \mathrm{~nm}, \sigma$ $\left.=2.22 \times 10^{-16} \mathrm{~cm}^{2}\right)$ and the photon density of the irradiation light that a DsRed molecule absorbs a photon each $40 \mu$ s and each $5.5 \mathrm{~ms}$ when irradiated with the focused beam $\left(40 \mathrm{~W} / \mathrm{cm}^{2}\right)$ and the wide beam $\left(300 \mathrm{~mW} / \mathrm{cm}^{2}\right)$, respectively. Therefore, the lifetime of the intermediate is estimated to be far less than milliseconds but in the microsecond time range. This model can also interpret the photoconversion observed for 532-nm nanosecond-pulsed laser irradiation. Here, a DsRed molecule absorbs a photon each $0.4-1.8 \mathrm{~ns}$ when irradiated with a pulsed $532-\mathrm{nm}$ laser at the irradiation power of $350-80 \mathrm{~mW} / \mathrm{cm}^{2}$, which is far shorter than the estimated lifetime of the intermediate. Since the pulse width of the irradiation laser $(8 \mathrm{~ns})$ allows a DsRed molecule to absorb two photons within one pulse, the conversion would occur within a pulse. Under such high photon

(37) Cotlet, M.; Habuchi, S.; Dirix, G.; Michiels, J.; Vanderleyden, J.; Lukyanov, K. A.; De Schryver, F. C.; Hofkens, J. In preparation. 


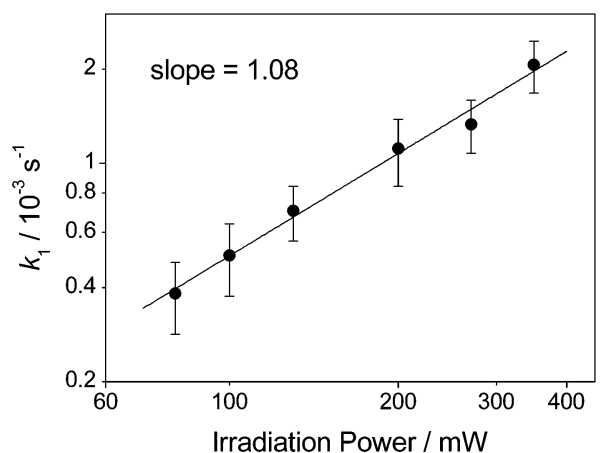

Figure 7. Double-logarithmic plot of the rate constants of the photoconversion from $\mathrm{R}$ to $\mathrm{SR}$.

Table 1. Light Source Dependence for the Rate of Photoconversion in DsRed

\begin{tabular}{lll}
\hline \multicolumn{1}{c}{ light source } & \multicolumn{1}{c}{ rate constant $\left(\mathrm{s}^{-1}\right)$} & relative rate $^{\mathrm{a}}$ \\
\hline nanosecond-pulsed 532 nm & $2.1 \times 10^{-3}$ & 1 \\
CW 568 nm (focused beam) & $3.9 \times 10^{-5}$ & 0.032 \\
CW 568 nm (wide beam) & $\mathrm{nd}^{b}$ & $\mathrm{nd}^{b}$ \\
\hline
\end{tabular}

${ }^{a}$ Relative rates were estimated by assuming a linear dependence of the rate constants on the excitation power. ${ }^{b}$ Change in the absorption spectrum is too small to determine the rate constant.

flux, most of the molecules are excited into the $S_{1}$ state and form a constant population of the intermediate independent of the excitation power. Hence, the absorption of the second photon would be quasi one-photon process, leading to the linear dependence of the rate constant of the conversion on the irradiation power. ${ }^{38}$ As expected, the rate constant of the conversion from $\mathrm{R}$ to $\mathrm{SR}\left(k_{1}\right)$ decreased linearly with reduction of the laser power (slope $=1.08$ ) on irradiation with the 532nm nanosecond-pulsed laser (see Figure 7).

The efficiency of the photoconversion on irradiation with the two light sources (532-nm nanosecond-pulsed laser, 568-nm CW laser) is summarized in Table 1.

The light source dependence of the photoconversion points to the fact that the intermediate species is formed within $8 \mathrm{~ns}$ from the $\mathrm{S}_{1}$ state of $\mathrm{R}$ and the lifetime of the state is in the order of microseconds. Although the nature of the intermediate is not clear at the moment, possible candidates are for example the triplet state, an isomerized form, or a different ionic state. These states could be formed within nanoseconds and could be stable for microseconds. Taking into account the proposed consecutive two-photon excitation mechanism, the conversion induced by 532-nm nanosecond-pulsed light and 568-nm focused CW light might take place via a higher excited state of the intermediate which is formed by excitation of the intermediate state. The hypothesis is supported by the fact that higher excited states often open new photophysical pathways leading to an efficient photochemical reaction. ${ }^{38-40}$

Photoinduced decarboxylation of wt-GFP has been thought to occur via a Kolbe-type reaction mechanism. This reaction is described as a photoinduced electron transfer from the adjacent glutamic acid to the chromophore which is accompanied by decarboxylation of the glutamic acid, followed by back electron

(38) Miyasaka, H.; Murakami, M.; Itaya, A.; Guillaumont, D.; Nakamura, S.; Irie, M. J. Am. Chem. Soc. 2001, 123, 753-754.

(39) Murakami, M.; Miyasaka, H.; Okada, T.; Kobatake, S.; Irie, M. J. Am. Chem. Soc. 2004, 126, 14764-14772.

(40) Tinnefeld, P.; Hofkens, J.; Herten, D. P.; Masuo, S.; Vosch, T.; Cotlet, M.; Habuchi, S.; Müllen, K.; De Schryver, F. C.; Sauer, M. ChemPhysChem 2004, 5, 1786-1790. transfer from the chromophore to the glutamic acid. ${ }^{8,9}$ The photoconversion of DsRed is accompanied by decarboxylation, most probably at the E215 residue, which points to the same type of reaction as that in wt-GFP. Decarboxylation in wt-GFP, however, results in the conversion from the protonated to the deprotonated form, and the cis conformation of the chromophore is kept. ${ }^{8}$ This is in contrast with the conversion of DsRed in which cis-trans isomerization of the chromophore is suggested to occur. In the case of wt-GFP, glutamate E222 (corresponds to E215 in DsRed) is incorporated into the hydrogen bonding network around the chromophore when the chromophore is protonated. Decarboxylation of E222 will, therefore, result in rearrangement of the network, and the deprotonated form of the chromophore will be more stabilized with this network. On the other hand, the chromophore of DsRed is deprotonated in the $\mathrm{R}$ form, and E215 does not interact directly with the chromophore by the hydrogen bonding network. ${ }^{36} \mathrm{X}$-ray crystallographic study also revealed that the deprotonated form of the DsRed chromophore is stabilized by a number of hydrogen bonds as well as a charge-charge interaction with a neighboring lysine residue (K163). ${ }^{36}$ Therefore, decarboxylation of E215 provides free volume around the chromophore which allows the chromophore to have a trans conformation as mentioned above, rather than modifying the protonation state.

Origin of the Change in the Fluorescence Spectrum on Irradiation. As shown in Figure 2a, the ratio of the green to red emission (470-nm excitation) has changed from 0.11 up to 17 upon irradiation with a 532-nm nanosecond-pulsed laser. The mechanism of the photoconversion demonstrated above offers factors contributing to the change in that ratio. The red emission excited at $470 \mathrm{~nm}$ occurs mainly via intratetramer energy transfer from $\mathrm{G}$ whose population stays constant on irradiation. An efficient Förster-type resonance energy transfer is expected between both $\mathrm{G}$ and $\mathrm{R}$ and $\mathrm{G}$ and $\mathrm{SR}$ due to the large spectral overlap (Förster distance is estimated to be 4.2 and $3.9 \mathrm{~nm}$ for energy transfer from $G$ to $R$ and $G$ to $S R$, respectively). However, R contributes dominantly to the red emission due to the dimness of SR ( $\Phi=0.012)$. Consequently, the decrease of the red fluorescence is the result of a decrease of the population of the $\mathrm{R}$ form, i.e., the conversion from $\mathrm{R}$ to $\mathrm{SR}$ and $\mathrm{R}$ to $\mathrm{B}$. Indeed, the time evolution of the intensity of the red emission is similar to the time evolution of the absorption of $\mathrm{R}$ (see Figure 2a inset). The increase of the fluorescence intensity of $\mathrm{G}$ is due to the conversion from $\mathrm{R}$ and $\mathrm{SR}$ to $\mathrm{B}$. Both of the conversions lead to a decrease in the population of the acceptor within the tetramer, resulting in the decrease in the efficiency of energy transfer from G to R or SR. Actually, the time evolution of the intensity of the green emission shows a similar behavior as the time evolution of the absorption of B (see Figure $2 \mathrm{a}$ inset).

Application of the Photoconversion for Selective Imaging. The change in the ratio of the green to red emission upon irradiation indicates the applicability of the photoconversion for selective imaging. Figure 8 shows dual-color fluorescence images of E. coli cells transfected with DsRed.

While the red emission was dominant before irradiation (see Figure 8a, b), the green emission revealed an enhancement of the intensity up to $250 \%$ and the red emission disappeared completely upon irradiation with a $543 \mathrm{~nm}$ CW laser (see Figure $8 \mathrm{c}, \mathrm{d})$. The changes in the intensity are similar to that observed for a pulsed laser irradiation (see Figure 2a). Although Marchant 

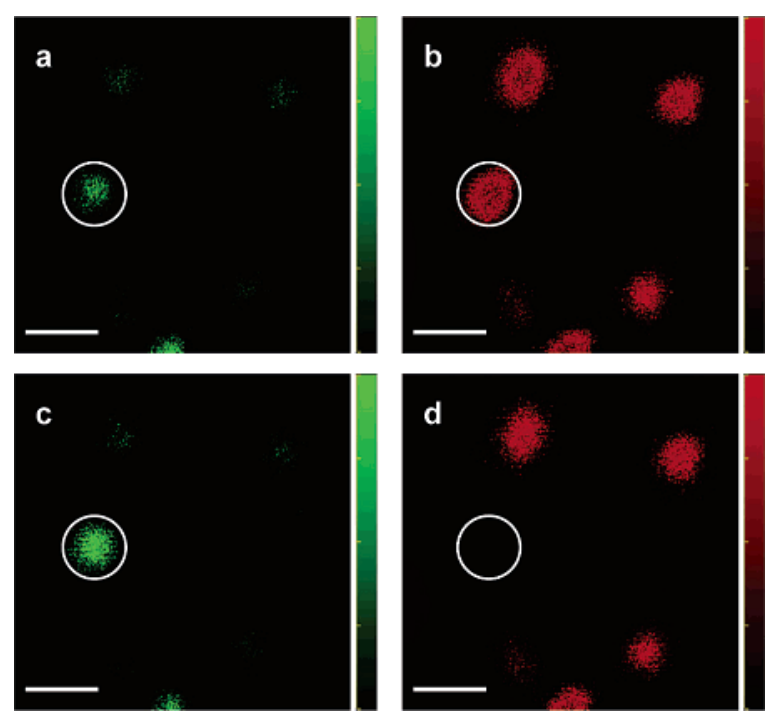

Figure 8. Dual color fluorescence images of $E$. coli cells transfected with DsRed excited at $488 \mathrm{~nm}$. (a, c) and (b, d) show the fluorescence images of the green and the red emission, respectively. (a) and (b) show the images before irradiation. (c) and (d) show the images after irradiation with a 543$\mathrm{nm} \mathrm{cw}$ laser. The irradiated cell is indicated by white circles. Scale bar $=$ $2 \mu \mathrm{m}$.

et al. reported about a similar photoconversion of DsRed, ${ }^{4,41}$ they used a femtosecond pulsed laser to induce the conversion via a multiphoton excitation. In our case, the photon density required for the conversion can be easily obtained with the $\mathrm{CW}$ lasers that are nowadays often used for life cell imaging. Even relatively low powers $(28 \mu \mathrm{W})$ are sufficient for conversion and optical marking, provided that high numerical objective lenses are used. Moreover, the photoconversion can be localized in three dimensions using focused CW laser light, since the high powers required for conversion with a $\mathrm{CW}$ laser are only reached in the focal point of the excitation beam, exploiting the dependence of the conversion efficiency on the photon flux.

\section{Concluding Remarks}

In the present study we investigated the mechanism of the photoconversion of DsRed on irradiation with a nanosecond-

(41) Robinson, L. C.; Marchant, J. S. Biophys. J. 2005, 88, 1444-1457. pulsed 532-nm light. It was clearly demonstrated that the different spectroscopic properties of the photoconverted species are related to the structure of the chromophore. It was suggested that the DsRed chromophore in the SR state has a trans configuration in which the hydrogen-bonding network around the chromophore is different from that in the $\mathrm{R}$ state. Furthermore, a glutamate located in close proximity to the chromophore (E215), is decarboxylated in SR which provides free volume around the chromophore. The dimness of SR $(\Phi=0.012)$ might, therefore, be attributed to the low activation energy to form the twisted configuration of the chromophore in the excited state which enhances the efficiency of the nonradiative deactivation. Decarboxylation of E215 on irradiation indicates a Kolbe-type reaction mechanism of the photoconversion from $\mathrm{R}$ to $\mathrm{SR}$ similarly observed before in wt-GFP. A consecutive two-photon excitation process, which probably results in a higher excited state of the chromophore, was proposed for this reaction upon irradiation with a 532-nm nanosecond-pulsed laser. The applicability of the photoconversion as a tool of selective imaging was proved by dual-color fluorescence imaging of DsRed transfected cells.

Finally, our results show the importance of the chromophore conformation for the brightness of the fluorescent protein. We also demonstrated the importance of the photon flux to control the photoconversion process. Although the control of the brightness of GFP-like proteins is still not straightforward yet, the detailed knowledge about the photoinduced processes in fluorescent proteins, as demonstrated here, can be used to engineer reliable photoactivatable fluorescent proteins and hence new imaging schemes.

Acknowledgment. S.Habuchi thanks JSPS for a postdoctoral fellowship. The KULeuven research fund (IDO), the Federal Science Policy through IAP/V/03, the Flemish Ministry of Education (GOA 2/01), the FWO, the FSR (UCL), and the FRFC (9.4532.04) are acknowledged for supporting this research.

Supporting Information Available: Detailed fitting of Figure 1 , full reference (PDF). This material is available free of charge via the Internet at http://pubs.acs.org.

JA047023O 